\title{
PAX8 expression reliably distinguishes pancreatic well-differentiated neuroendocrine tumors from ileal and pulmonary well-differentiated neuroendocrine tumors and pancreatic acinar cell carcinoma
}

\author{
Ankur R Sangoi ${ }^{1,2,4}$, Robert S Ohgami ${ }^{2,4}$, Rish K Pai ${ }^{3}$, Andrew H Beck ${ }^{2}$, \\ Jesse K McKenney ${ }^{2}$ and Reetesh K Pai ${ }^{2}$ \\ ${ }^{1}$ Department of Pathology, El Camino Hospital, Mountain View, CA, USA; ${ }^{2}$ Department of Pathology, \\ Stanford University, Stanford, CA, USA and ${ }^{3}$ Department of Pathology, Washington University, \\ St Louis, MO, USA
}

\begin{abstract}
PAX (paired box) genes encode a family of transcription factors that regulate organogenesis in a variety of organs. Very little is known about the role of PAX8 in endocrine cell development and the expression of PAX8 in neuroendocrine tumors. The purpose of this study was to analyze PAX8 immunohistochemical expression in gastroenteropancreatic and pulmonary well-differentiated neuroendocrine tumors to determine whether PAX8 can reliably distinguish pancreatic neuroendocrine tumors from neuroendocrine tumors of other anatomic sites and other pancreatic non-ductal neoplasms. In total, 221 well-differentiated neuroendocrine tumors were evaluated: 174 primary neuroendocrine tumors (66 pancreatic, 31 ileal, 21 pulmonary, 20 gastric, 17 rectal, 11 appendiceal, and 8 duodenal) and 47 neuroendocrine tumors metastatic to the liver (31 pancreatic, 11 ileal, 2 pulmonary, 2 duodenal, and 1 rectal). Fifteen solid-pseudopapillary neoplasms and six acinar cell carcinomas of the pancreas were also evaluated. PAX8 was positive in 49/66 (74\%) primary pancreatic neuroendocrine tumors. PAX8 expression did not correlate with World Health Organization categorization, grade, size, functional status, or the presence of liver or lymph node metastasis. PAX8 expression was identified in $0 / 31(0 \%)$ ileal, 0/21 (0\%) pulmonary, $2 / 20(10 \%)$ gastric, $5 / 17(29 \%)$ rectal, $1 / 11(9 \%)$ appendiceal, and $6 / 8(75 \%)$ duodenal neuroendocrine tumors. PAX8 was positive in 4/15 (27\%) solid-pseudopapillary neoplasms of the pancreas, whereas all acinar cell carcinomas (0/6) lacked immunoreactivity. Among liver metastases, only pancreatic neuroendocrine tumors $(20 / 31,65 \%)$ were PAX8 positive, whereas no cases of ileal (0/11), pulmonary (0/2), duodenal (0/2), and rectal (0/1) neuroendocrine tumor metastases were PAX8 positive. PAX8 is expressed in primary and metastatic pancreatic well-differentiated neuroendocrine tumors, and its expression can reliably distinguish pancreatic from ileal and pulmonary well-differentiated neuroendocrine tumors. Duodenal neuroendocrine tumors and a subset of rectal, gastric, and appendiceal neuroendocrine tumors may also express PAX8. PAX8 expression can distinguish pancreatic neuroendocrine tumors from acinar cell carcinomas, but its utility in distinguishing neuroendocrine tumors from solid-pseudopapillary neoplasms is limited.
\end{abstract} Modern Pathology (2011) 24, 412-424; doi:10.1038/modpathol.2010.176; published online 1 October 2010

Keywords: carcinoid; endocrine tumor; immunohistochemistry; neuroendocrine tumor; pancreas; pancreatic; PAX8

Correspondence: Dr Reetesh K Pai, MD, Department of Pathology, Stanford University, 300 Pasteur Drive, Room H-2110, Stanford, CA 94305, USA.

E-mail: reeteshp@stanford.edu

${ }^{4}$ These two authors are the co-first authors.

Received 18 May 2010; revised 1 July 2010; accepted 11 July 2010; published online 1 October 2010
Well-differentiated neuroendocrine tumors are a diverse family of neoplasms with varied clinical behavior. $^{1}$ Although the incidence of neuroendocrine tumors has been reported as relatively low (2.5-5 cases per 100000), their prevalence has increased substantially in recent years. ${ }^{2-5}$ 
Thus, given the increased significance of these tumors, a more detailed understanding of these neoplasms is critical. Neuroendocrine tumors of gastrointestinal, pulmonary, and pancreatic origin exhibit similar histologic features and cannot be distinguished from each other by morphology alone. Furthermore, the classic immunohistochemical neuroendocrine markers synaptophysin and chromogranin are broadly expressed in nearly all neuroendocrine tumors, and thus do not distinguish a tumor from one site vs another. In addition, because conventional radiological methods and even computed tomography or magnetic resonance imaging studies can suffer from relatively low sensitivity and specificity for determining a site of origin, developing new modalities for identifying the primary origin of a neuroendocrine tumor is critical. ${ }^{6,7}$

In recent years, several studies have used singleimmunohistochemical markers as well as panels of several markers to delineate a site of origin for metastatic neuroendocrine tumor. For instance, Schmitt et al, identified Islet-1 as a marker to differentiate neuroendocrine tumors of pancreatic origin from those of other sites, whereas other studies have used TTF-1 and CDX-2 for identifying pulmonary $v s$ gastrointestinal origin. ${ }^{8-12}$ Recently, Srivastava et $a l^{13}$ demonstrated that a panel of antibodies against PDX-1, CDX-2, neuroendocrine secretory protein-55 (NESP-55), and TTF-1 can distinguish pancreatic from pulmonary carcinoid tumors. However, at present, no single-immunohistochemical marker has been identified that reliably differentiates pancreatic neuroendocrine tumors from tumors of other sites within the gastrointestinal and pulmonary tracts.

$P A X$ (paired box) genes encode a family of transcription factors that are expressed in embryogenesis and regulate organogenesis in a variety of organs. ${ }^{14,15}$ In particular, $P A X 4$ and $P A X 6$ are essential for development of pancreatic islet cells as well as endocrine cell development in the small intestine and stomach. ${ }^{14-16}$ Although $P A X 8$ has been most studied in renal tissues and neoplasms, ${ }^{17}$ it has been recently recognized as a useful marker in distinguishing ovarian from breast carcinoma, ${ }^{18}$ and as a marker of thyroid epithelial neoplasms. ${ }^{19}$ Moreover, our group recently observed intense PAX8 reactivity in pancreatic neuroendocrine tumors and normal pancreatic islets in a large tissue microarray evaluation of PAX8 immunoreactivity of non-renal tissues/neoplasms. ${ }^{20}$ Very little is known about the role of $P A X 8$ in endocrine cell development, and differential PAX8 expression in neuroendocrine tumors of the pancreas, gastrointestinal tract, and lung could be of potential clinical utility. Herein, we analyze PAX8 immunohistochemical expression in gastroenteropancreatic and pulmonary welldifferentiated neuroendocrine tumors to (1) determine whether PAX8 can reliably distinguish pancreatic neuroendocrine tumors from neuroendocrine tumors of other anatomic sites and other pancreatic epithelial neoplasms (ie, solid-pseudopapillary neoplasm, and acinar cell carcinoma) and (2) examine whether PAX8 expression is retained in metastases from well-differentiated neuroendocrine tumors.

\section{Materials and methods}

\section{Study Group}

A total of 221 neuroendocrine tumor specimens from 197 patients comprise this study and were identified from a search of the surgical pathology database of Stanford University Medical Center between the years 2004 and 2009 for primary well-differentiated pancreatic, gastrointestinal and pulmonary neuroendocrine tumors, and metastatic well-differentiated neuroendocrine tumors to the liver. A total of 174 primary neuroendocrine tumors were included and comprised 66 pancreatic, 31 ileal, 21 pulmonary, 20 gastric, 17 rectal, 11 appendiceal, and 8 duodenal neuroendocrine tumors. All 66 primary pancreatic neuroendocrine tumors were surgical resection specimens. For neuroendocrine tumors from non-pancreatic sites, both small tissue biopsy $(n=36)$ and surgical resection $(n=72)$ specimens were included. Fortyseven metastatic neuroendocrine tumors to the liver were included for which the primary site of origin was the pancreas (31), ileum (11), lung (2), duodenum (2), and rectum (1). Of the 47 metastatic neuroendocrine tumors, both needle core biopsy $(n=5)$ and surgical resection $(n=42)$ specimens were included. Pairs of primary and metastatic tumor tissue were available in 25 of these patients. In addition, surgical resections of 15 solid-pseudopapillary neoplasms of the pancreas and 6 acinar cell carcinomas of the pancreas identified from a search of the surgical pathology databases of both Stanford University Medical Center and Washington University between the years 2000 and 2009 were included in this study.

All primary pancreatic neuroendocrine tumors were classified into three groups according to the World Health Organization (WHO) criteria and also classified using a published two-tiered classification scheme (Hochwald et al: low grade: < 2 mitoses/50 high-powered fields (HPF) and no necrosis; and intermediate grade: $\geq 2$ mitosis/50 $\mathrm{HPF}$ and/or necrosis). ${ }^{21,22}$ The functional status of pancreatic neuroendocrine tumors was determined according to clinical and serologic findings at presentation. Neuroendocrine tumors from the gastrointestinal tract and lung were also classified using WHO criteria. ${ }^{23}$

\section{Histologic Evaluation and PAX8 Immunohistochemistry}

All hematoxylin and eosin (H\&E)-stained sections and relevant immunostains were reviewed to confirm 
diagnoses. Initially, all tissues were fixed in $10 \%$ neutral-buffered formalin, dehydrated in graded alcohols, cleared in xylene, and embedded in paraffin. Whole tumor sections for immunohistochemistry were cut at $4 \mathrm{~mm}$, deparaffinized in xylene, and rehydrated through graded alcohols to distilled water before undergoing antigen retrieval by heat treatment in EDTA solution ( $\mathrm{pH}$ 8.0, 20 min). An automated detection using a Ventana Benchmark XT Autostainer (Tuscon, AZ, USA) was used with 1:40 dilution of anti-PAX8 (polyclonal; Proteintech, Chicago, IL, USA). Positive external controls were stained in parallel with study group tissues, consisting of sections from paraffin-embedded clear cell renal cell carcinoma from a radical nephrectomy specimen, in which diffuse nuclear immunoreactivity for PAX8 was present in the tumor cells. B-lymphocytes within tissue sections also served as positive internal controls. ${ }^{20,24}$

The extent of PAX8 nuclear staining was jointly evaluated by two investigators (R.S.O. and R.K.P.), and the results were tabulated semiquantitatively as follows: $1+$ staining in $5-25 \%$ of cells; $2+$ staining in $26-75 \%$ of cells; and $3+$ staining in $>75 \%$ of cells. The intensity of PAX8 nuclear labeling was qualitatively evaluated as either strong (defined as nuclear reactivity easily visible at $\times 40$ magnification) or weak (defined as nuclear reactivity visible at $\times 100$ or $\times 200$ magnification but not clearly present at lower magnification). Cytoplasmic staining in the absence of nuclear labeling was recorded but scored as negative.

Fisher's exact test (two tailed), $\chi^{2}$, or Student's $t$-tests were used to evaluate the correlation PAX8 immunoreactivity and clinicopathologic features of pancreatic neuroendocrine tumors. The significance level was set at $P<0.05$.

\section{Results}

\section{PAX8 Immunohistochemistry}

In all pancreatic neuroendocrine tumors analyzed, adjacent non-neoplastic islet cells demonstrated diffuse, strong labeling with PAX8, whereas normal acinar and ductal epithelial cells were negative (Figure 1). The staining pattern of well-differentiated pancreatic neuroendocrine tumors included in this study is summarized in Table 1. Overall, $49 / 66(74 \%)$ of pancreatic neuroendocrine tumors were positive for PAX8. Among these, the majority $(37 / 49, \quad 76 \%)$ showed $3+(>75 \%)$ staining (Figure 1). Of the positive cases, most (39/49, 78\%) demonstrated a strong nuclear staining intensity. Cytoplasmic staining without nuclear labeling was rare in pancreatic neuroendocrine tumors and identified in only 4/66 (6\%) cases.

Compared with pancreatic neuroendocrine tumors, well-differentiated neuroendocrine tumors from all other anatomic sites demonstrated significantly diminished PAX8 reactivity (14/108, 13\%)
$(P<0.0001)$ (Table 2). PAX8 labeling varied depending on anatomic location of the neuroendocrine tumor and was more frequently detected in duodenal $(6 / 8,75 \%)$ and rectal $(5 / 17,29 \%)$ neuroendocrine tumors (Figure 2), with tumors from both sites demonstrating $3+$ extent of staining (5/6 and $4 / 5$ cases, respectively). However, duodenal neuroendocrine tumors more often had strong nuclear staining intensity (6/6 cases) compared with rectal neuroendocrine tumors, which typically displayed weak nuclear labeling (4/5 cases). PAX8 was infrequently positive in gastric $(2 / 19,11 \%)$ and appendiceal (1/11, 9\%) neuroendocrine tumors. Importantly, PAX8 expression was absent in ileal $(0 / 31)$ and pulmonary (0/21) neuroendocrine tumors. Cytoplasmic staining without nuclear labeling was noted in a subset of gastrointestinal and pulmonary neuroendocrine tumors 19/104 (18\%). Rectal neuroendocrine tumors most commonly demonstrated cytoplasmic reactivity without nuclear labeling (10/17 cases, $59 \%$ ) following by gastric neuroendocrine tumors $(5 / 20,25 \%)$ and pulmonary neuroendocrine tumors (4/21, 19\%) (Figure 2). Neuroendocrine tumors from the ileum, appendix, and duodenum did not display cytoplasmic staining without nuclear labeling.

Twenty of the $47(43 \%)$ metastatic well-differentiated neuroendocrine tumors to the liver were positive for PAX8 (Table 3), with all positive cases representing pancreatic primary tumors (Figure 3). All metastases from ileal, pulmonary, duodenal, and rectal primary well-differentiated neuroendocrine tumors were negative for PAX8. The immunohistochemical staining pattern in matched primary and metastatic tumor deposits correlated in all nine cases of ileal neuroendocrine tumors and in 13 of 16 $(81 \%)$ pancreatic neuroendocrine tumors. In two cases of pancreatic neuroendocrine tumors, the primary tumor demonstrated $3+$, strong staining, whereas the metastatic tumor deposit in the liver lacked PAX8 immunoreactivity. In one case of pancreatic neuroendocrine tumor, the primary tumor was PAX8 negative, whereas the metastatic tumor deposit was diffusely and strongly PAX8 positive (Figure 3).

Four of the 15 solid-pseudopapillary neoplasms $(4 / 15,27 \%)$ demonstrated PAX8 labeling, and the extent of staining was $1+$ ( 1 case) or $2+$ ( 3 cases $)$ (Table 3). The labeling in three of four cases was weak, whereas strong nuclear labeling was observed in one case that exhibited $2+$ extent of staining (Figure 4). The remainder of solid-pseudopapillary neoplasms of the pancreas lacked either nuclear or cytoplasmic staining with PAX8. All six acinar cell carcinomas were negative for PAX8.

\section{Correlation of Clinicopathologic Features and PAX8 Immunohistochemistry}

The clinicopathologic features of the pancreatic neuroendocrine tumors included in this study are 


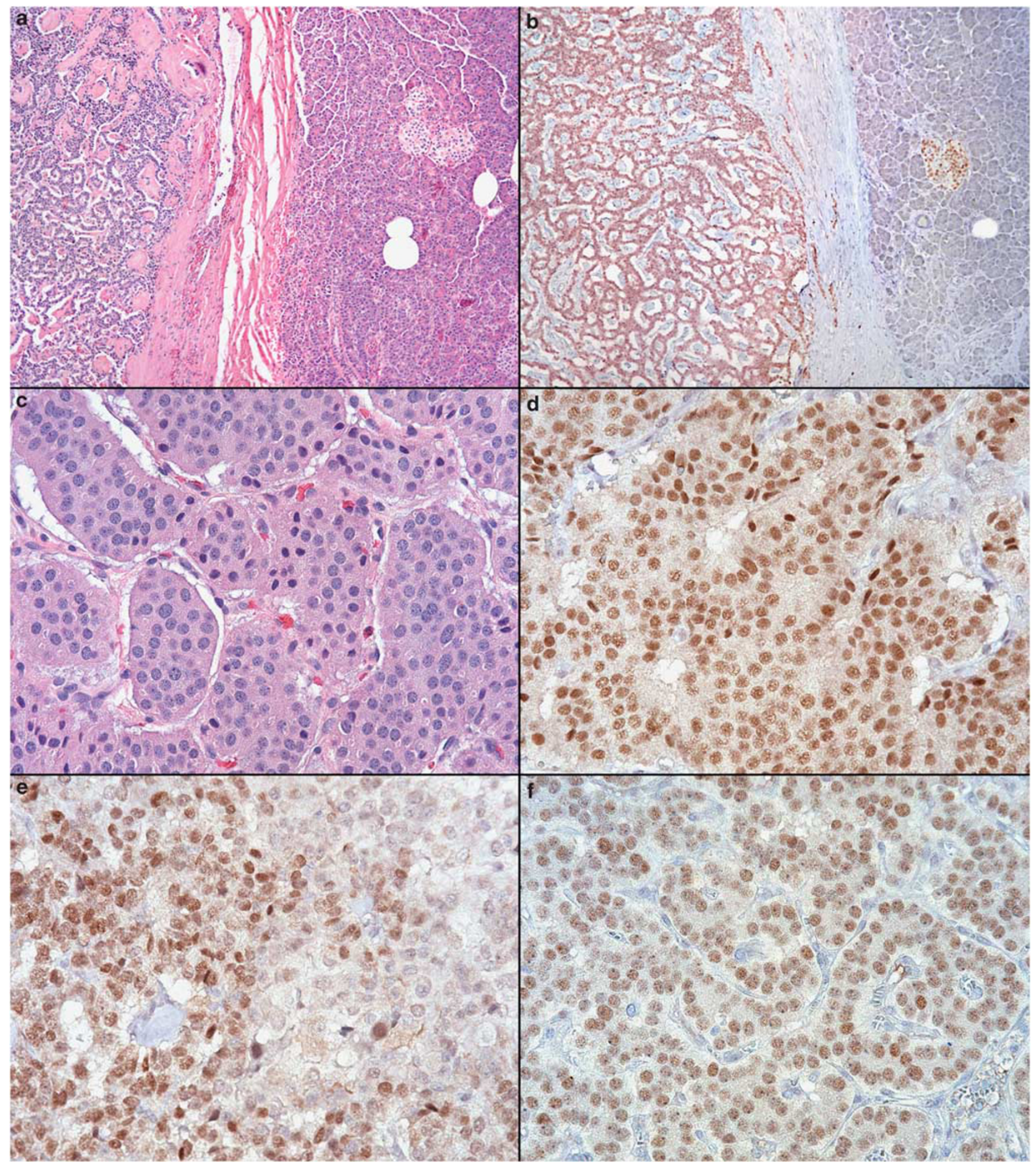

Figure 1 (a) Low-power image of well-differentiated pancreatic neuroendocrine tumor (H\&E, $\times 200)$, with adjacent non-neoplastic pancreas. (b) $3+$, strong PAX8 staining in well-differentiated pancreatic neuroendocrine tumor and adjacent islet cells $(\times 200)$. (c) Well-differentiated pancreatic neuroendocrine tumor $(\mathrm{H} \& \mathrm{E}, \times 400)$ demonstrating $3+$, strong PAX8 nuclear staining $(\mathbf{d}$; $\times 400)$. (e) Well-differentiated pancreatic neuroendocrine tumor showing $2+$, strong PAX8 nuclear staining $(\times 400)$. (f) Well-differentiated pancreatic neuroendocrine tumor showing $3+$, weak PAX8 staining $(\times 400)$.

summarized in Table 4. Patient ages for pancreatic neuroendocrine tumors ranged from 36 to 82 years (mean 57 years), with a near-equal sex distribution (36 males and 30 females). Most patients (46/66, 70\%) underwent a distal pancreatectomy and splenectomy, as their tumors were located in the body or tail of the pancreas. Twenty-two patients had clinical evidence of liver metastasis either at the time of tumor 
Table 1 PAX8 immunoreactivity in pancreatic neuroendocrine tumors

\begin{tabular}{|c|c|c|c|c|c|c|}
\hline \multirow[t]{3}{*}{ Feature } & \multirow[t]{3}{*}{ No. of cases } & \multicolumn{5}{|c|}{ PAX8 immunoreactivity } \\
\hline & & \multirow{2}{*}{$\begin{array}{l}\text { Total no. positive } \\
\text { (\% of total) }\end{array}$} & \multicolumn{3}{|c|}{ Extent of staining } & \multirow{2}{*}{$\begin{array}{l}\text { No. of cases with } \\
\text { strong staining } \\
\text { intensity (\% of pos }\end{array}$} \\
\hline & & & No. $3+$ & No. $2+$ & No. $1+$ & \\
\hline Overall & 66 & $49(74)$ & 37 & 7 & 5 & $39(78)$ \\
\hline \multicolumn{7}{|l|}{ WHO category } \\
\hline $1 \mathrm{a}$ & 21 & $18(27)$ & 14 & 4 & 0 & $15(83)$ \\
\hline $1 \mathrm{~b}$ & 17 & $12(18)$ & 9 & 1 & 2 & 8 (67) \\
\hline 2 & 28 & $19(29)$ & 14 & 2 & 3 & $16(84)$ \\
\hline \multicolumn{7}{|l|}{ Hochwald et al grade } \\
\hline Low & 53 & $42(64)$ & 31 & 6 & 5 & $34(81)$ \\
\hline Intermediate & 13 & $7(10)$ & 6 & 1 & 0 & $5(71)$ \\
\hline \multicolumn{7}{|l|}{ Location } \\
\hline Head & 20 & $14(21)$ & 8 & 2 & 4 & $11(79)$ \\
\hline Body or tail & 46 & $35(53)$ & 29 & 5 & 1 & $28(80)$ \\
\hline Functional clinically & 14 & $11(17)$ & 8 & 2 & 1 & \\
\hline Insulin producing & 12 & $10(15)$ & 8 & 1 & 1 & $8(80)$ \\
\hline Gastrin producing & 1 & 0 & 0 & 0 & 0 & 0 \\
\hline Glucagon producing & 1 & $1(2)$ & 0 & 1 & 0 & $1(100)$ \\
\hline Non-functional & 52 & $38(58)$ & 29 & 5 & 4 & $30(79)$ \\
\hline \multicolumn{7}{|l|}{ Metastatic disease } \\
\hline Absent & 37 & $29(44)$ & 22 & 5 & 2 & 22 \\
\hline Present & 29 & $20(30)$ & 15 & 2 & 3 & 17 \\
\hline Liver & 22 & $16(24)$ & 11 & 2 & 3 & 13 \\
\hline Lymph node & 20 & $12(18)$ & 12 & 0 & 0 & 10 \\
\hline Both liver and lymph node & 11 & $6(9)$ & 6 & 0 & 0 & 6 \\
\hline
\end{tabular}

Extent of staining scored as follows: $1+, 5-25 \%$ tumor nuclei; $2+, 26-75 \%$ tumor nuclei; $3+,>75 \%$ tumor nuclei.

Intensity of staining scored as strong (easily visible at $\times 40$ magnification) vs weak (visible at $\times 100$ or $\times 200$ magnification but not clearly visible at low-power magnification).

Table 2 PAX8 immunoreactivity in gastroenteropancreatic/pulmonary neuroendocrine tumors and other non-neuroendocrine pancreatic tumors

\begin{tabular}{|c|c|c|c|c|c|c|}
\hline \multirow[t]{3}{*}{ Site/diagnosis } & \multirow[t]{3}{*}{ No. of cases } & \multicolumn{5}{|c|}{ PAX8 immunoreactivity } \\
\hline & & \multirow{2}{*}{$\begin{array}{c}\text { Total no. } \\
\text { positive (\%) }\end{array}$} & \multicolumn{3}{|c|}{ Extent of staining } & \multirow{2}{*}{$\begin{array}{l}\text { No. of cases with } \\
\text { strong staining } \\
\text { intensity ( } \% \text { of pos }\end{array}$} \\
\hline & & & No. $3+$ & No. $2+$ & No. $1+$ & \\
\hline \multicolumn{7}{|l|}{ Pulmonary } \\
\hline Typical carcinoid & 18 & 0 & 0 & 0 & 0 & 0 \\
\hline Atypical carcinoid & 3 & 0 & 0 & 0 & 0 & 0 \\
\hline Terminal ileum & 31 & 0 & 0 & 0 & 0 & 0 \\
\hline Stomach & 20 & $2(10)$ & 0 & 0 & 2 & 0 \\
\hline Rectum & 17 & $5(29)$ & 4 & 0 & 1 & 0 \\
\hline Appendix & 11 & $1(9)$ & 1 & 0 & 0 & $1(100)$ \\
\hline Duodenum & 8 & $6(75)$ & 5 & 0 & 1 & $6(100)$ \\
\hline Solid-pseudopapillary neoplasm & 15 & $4(27)$ & 0 & 3 & 1 & $1(25)$ \\
\hline Acinar cell carcinoma & 5 & 0 & 0 & 0 & 0 & 0 \\
\hline
\end{tabular}

Extent of staining scored as follows: $1+, 5-25 \%$ tumor nuclei; $2+, 26-75 \%$ tumor nuclei; $3+,>75 \%$ tumor nuclei.

Intensity of staining scored as strong (easily visible at $\times 40$ magnification) vs weak (visible at $\times 100$ or $\times 200$ magnification but not clearly visible at low-power magnification).

resection or following surgical resection; 19 of these patients had concurrent or subsequent surgical resection of the liver metastases. In two cases, a pancreatic neuroendocrine tumor was incidentally discovered following distal pancreatectomy for invasive ductal adenocarcinoma.

The correlation of clinicopathologic features and PAX8 expression in pancreatic neuroendocrine 
tumors is summarized in Table 4. PAX8 was not associated with WHO category $(P=0.22)$, location (head vs body/tail, $P=0.76$ ), functional status
$(P=1.0)$, or the presence of liver $(P=1.0)$ or lymph node metastases $(P=0.12)$. Although not statistically significant, there was a trend toward decreased

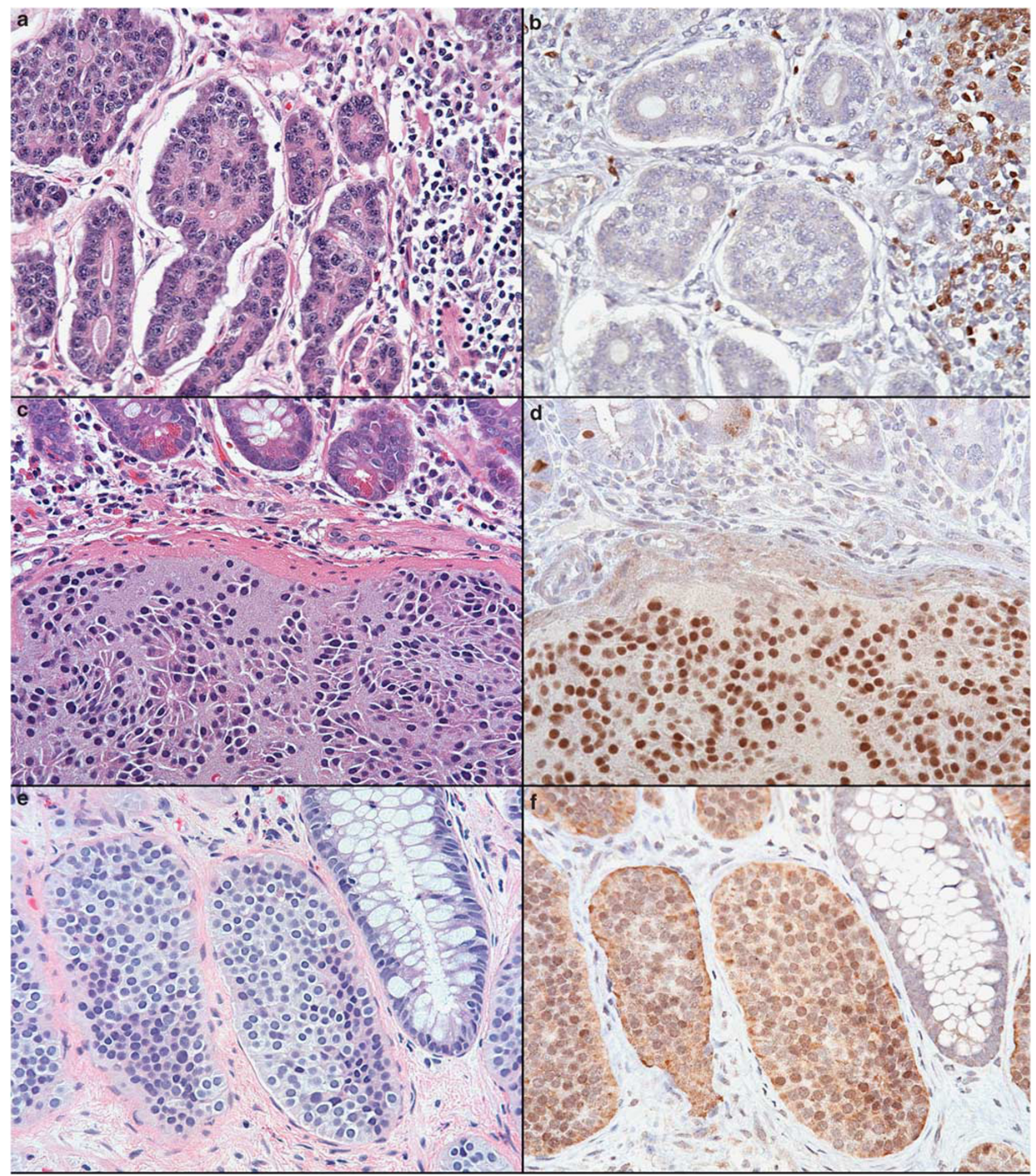

Figure 2 PAX8 immunohistochemical staining in primary gastrointestinal and pulmonary well-differentiated neuroendocrine tumors. $($ a) Ileal neuroendocrine tumor $(\mathrm{H} \& \mathrm{E}, \times 400)$ negative for PAX8 $(\mathbf{b} ; \times 400)$. Note PAX8-positive B-lymphocytes (right). (c) Duodenal neuroendocrine tumor $(\mathrm{H} \& \mathrm{E}, \times 400) 3+$, strong positive for PAX8 $(\mathbf{d} ; \times 400)$. Note the scattered positive neuroendocrine cells within the duodenal crypt epithelium. (e) Rectal neuroendocrine tumor $(\mathrm{H} \& \mathrm{E}, \times 400)$ with $3+$, weak nuclear and cytoplasmic PAX8-positive staining $(\mathbf{f} ; \times 400)$. (g) Rectal neuroendocrine tumor with weak cytoplasmic PAX8 staining without nuclear labeling $(\times 400)$. Note the strong staining of adjacent lymphoid aggregate. (h) Gastric neuroendocrine tumor with weak cytoplasmic PAX8 staining without nuclear labeling $(\times 400)$. 


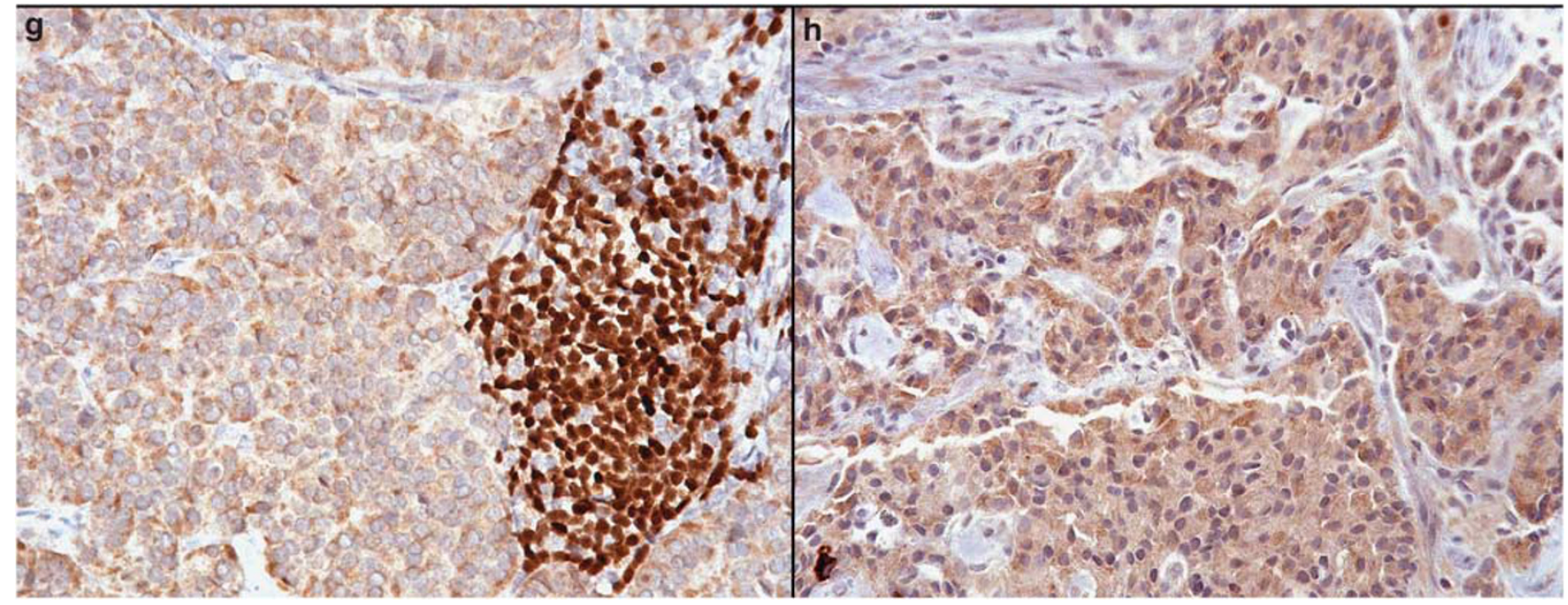

Figure 2 Continued.

Table 3 PAX8 immunoreactivity in metastatic neuroendocrine tumors to the liver

\begin{tabular}{|c|c|c|c|c|c|c|}
\hline \multirow[t]{3}{*}{ Primary site } & \multirow[t]{3}{*}{ No. of cases } & \multicolumn{4}{|c|}{ PAX8 immunoreactivity } & \multirow{3}{*}{$\begin{array}{l}\text { Correlation of PAX8 } \\
\text { staining in primary and } \\
\text { metastatic NET }(\%)\end{array}$} \\
\hline & & \multirow{2}{*}{$\begin{array}{c}\text { Total no. } \\
\text { positive }(\%)\end{array}$} & \multicolumn{3}{|c|}{ Extent of staining } & \\
\hline & & & No. $3+$ & No. $2+$ & No. $1+$ & \\
\hline Pancreas & 31 & $20(65)$ & 12 & 3 & 5 & $13 / 16(81)$ \\
\hline Ileum & 11 & 0 & 0 & 0 & 0 & 9/9 (100) \\
\hline Lung & 2 & 0 & 0 & 0 & 0 & 0 \\
\hline Duodenum & 2 & 0 & 0 & 0 & 0 & 0 \\
\hline Rectum & 1 & 0 & 0 & 0 & 0 & 0 \\
\hline
\end{tabular}

Extent of staining scored as follows: $1+, 5-25 \%$ tumor nuclei; $2+, 26-75 \%$ tumor nuclei; $3+,>75 \%$ tumor nuclei.

PAX8 expression for intermediate grade pancreatic neuroendocrine tumors $(P=0.08)$, as classified by the Hochwald et al grading scheme, as well as a trend toward increased tumor size in PAX8-negative pancreatic neuroendocrine tumors $(P=0.06)$.

\section{Discussion}

Well-differentiated neuroendocrine tumors are a diverse group of neoplasms that can arise from neuroendocrine cells present within virtually every organ in the body but are most commonly found in the pancreas, gastrointestinal tract, and bronchopulmonary system. Although quite rare compared with other carcinomas and comprising $<1 \%$ of all cancers, the prevalence of well-differentiated neuroendocrine tumors has increased over the last few decades., ${ }^{2,21,23,25}$ Although some well-differentiated neuroendocrine tumors secrete peptides and neuroamines leading to distinct clinical syndromes and early detection, many welldifferentiated neuroendocrine tumors are clinically silent until late presentation as metastatic disease, particularly hepatic metastases, with an unknown primary site of origin at presentation. ${ }^{2,6}$ At present, conventional imaging modalities are unable to accurately identify the primary site of tumor in $20-50 \%$ of cases. ${ }^{6}$ Histologically, well-differentiated neuroendocrine tumors from all anatomic sites of origin are indistinguishable, and it is not possible based on morphology alone to reliably predict the primary site of origin of a well-differentiated neuroendocrine tumor when confronted with a metastasis. The distinction between pancreatic neuroendocrine tumors and neuroendocrine tumors from the gastrointestinal and pulmonary tracts is becoming increasingly important in the therapeutic management of patients with metastatic disease. Recent clinical trials have demonstrated that patients with pancreatic neuroendocrine tumors more often benefit from temozolimide-based therapies compared with neuroendocrine tumors from non-pancreatic sites of origin. ${ }^{26,27}$ Thus, an immunohistochemical marker that can reliably determine the primary site of origin for a metastatic neuroendocrine tumor would have tremendous diagnostic utility. 


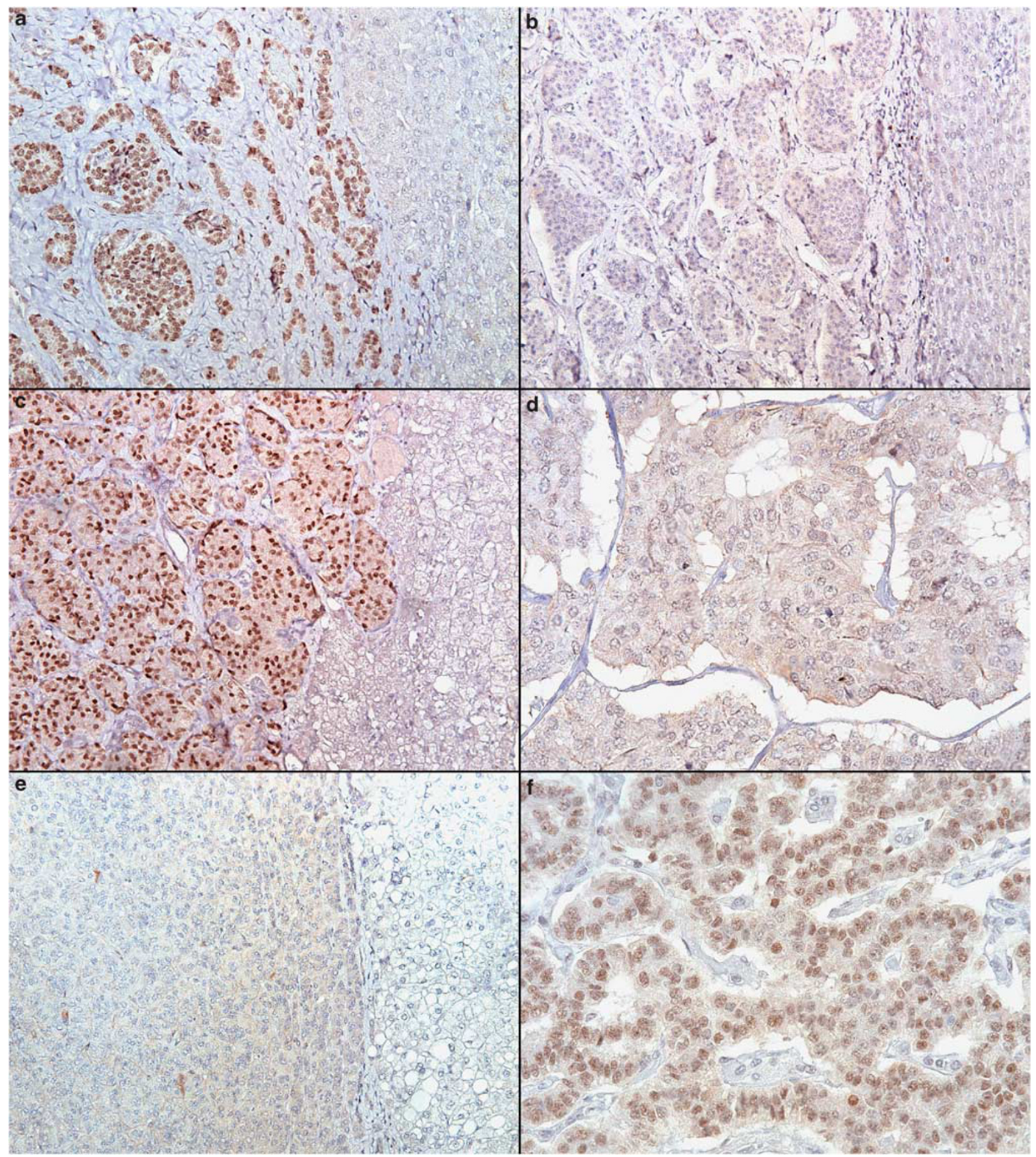

Figure 3 PAX8 immunohistochemical staining in metastatic well-differentiated neuroendocrine tumors to the liver. (a) Pancreatic neuroendocrine tumor with $3+$, strong PAX8 staining $(\times 200)$, with negative staining in adjacent non-neoplastic liver. (b) Ileal neuroendocrine tumor negative for PAX8 $(\times 200)$. (c) Pancreatic neuroendocrine tumor with $3+$, strong PAX8 staining in the liver metastasis $(\times 200)$ but negative PAX8 staining in the primary pancreatic tumor $(\mathbf{d} ; \times 400)$. (e) Pancreatic neuroendocrine tumor with negative PAX8 staining in the liver metastasis $(\times 200)$ but $3+$, weak PAX8 staining in the primary pancreatic tumor $(\mathbf{f} ; \times 400)$.

$P A X 8$ is a member of the $P A X$ (paired box) genes, which encode a family of transcription factors that regulate organogenesis. In the pancreas, PAX4 and $P A X 6$ gene expression is confined to endocrine precursor cells, suggesting that $P A X 4$ and $P A X 6$ are required during early steps to generate specific endocrine cells of the pancreas. ${ }^{16,28}$ Very little is known about the role of PAX8 in islet cell 


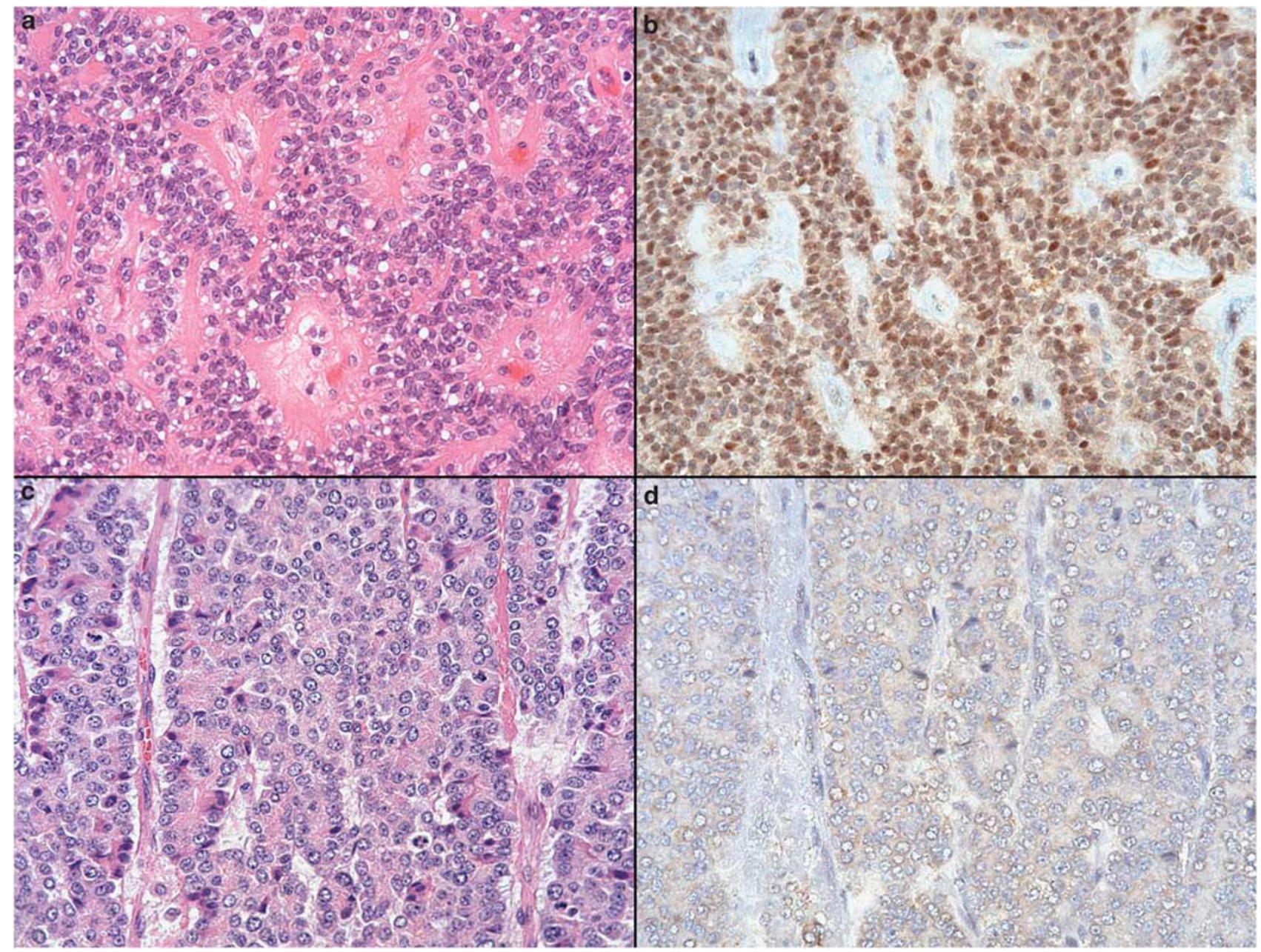

Figure 4 (a) Solid-pseudopapillary neoplasm of the pancreas $(\mathrm{H} \& \mathrm{E}, \times 400)$ demonstrating strong nuclear PAX8 staining $(\mathbf{b} ; \times 400)$ $(\mathbf{c})$ Acinar cell carcinoma of the pancreas $(\mathrm{H} \& \mathrm{E}, \times 400)$ with negative PAX8 staining $(\mathbf{d} ; \times 400)$.

Table 4 Correlation of clinicopathologic features and PAX8 immunoreactivity in pancreatic neuroendocrine tumors

\begin{tabular}{|c|c|c|c|}
\hline Clinicopathologic feature & $\begin{array}{c}\text { PAX8-positive } \\
\text { pancreatic NET } \\
\text { (n=49) }\end{array}$ & $\begin{array}{l}\text { PAX8-negative } \\
\text { pancreatic NET } \\
\quad(\mathrm{n}=17)\end{array}$ & P-value \\
\hline Median age (years) & 53 & 60 & 0.26 \\
\hline Gender (M:F) & $28: 21$ & $8: 9$ & 0.66 \\
\hline Mean tumor size (cm) & 3.0 & 5.1 & 0.06 \\
\hline Lymph node metastasis & $12 / 49(24 \%)$ & $8 / 17(47 \%)$ & 0.12 \\
\hline Liver metastasis & $16 / 49(33 \%)$ & $6 / 17(35 \%)$ & 1.0 \\
\hline WHO category & & & 0.22 (1a vs $1 \mathrm{~b}$ or 2$)$ \\
\hline $1 \mathrm{a}$ & $18(37 \%)$ & $3(18 \%)$ & \\
\hline $1 \mathrm{~b}$ & $12(24 \%)$ & $5(29 \%)$ & \\
\hline 2 & $19(39 \%)$ & $9(53 \%)$ & \\
\hline Hochwald et al grade & & & 0.08 \\
\hline Low grade & $42(86 \%)$ & $11(65 \%)$ & \\
\hline Intermediate grade & $7(14 \%)$ & $6(35 \%)$ & \\
\hline Location & & & 0.76 \\
\hline Head & 14 & 6 & \\
\hline Body/tail & 35 & 11 & \\
\hline
\end{tabular}


development. The expression of PAX8 in adjacent benign islet cells in all of our cases of pancreatic neuroendocrine tumors suggests that PAX8 expression is critical for islet cell function. We have also demonstrated PAX8 immunoreactivity in islet cells seen in the fetal pancreas early in gestation (data not shown), indicating that PAX8 expression occurs relatively early in pancreatic islet cell development.

Our study shows that PAX8 immunohistochemistry is strongly expressed in pancreatic neuroendocrine tumors and its metastases. In contrast, PAX8 was not observed in ileal or pulmonary well-differentiated neuroendocrine tumors, whereas other gastrointestinal neuroendocrine tumors, particularly those of the duodenum, stomach, and rectum, may demonstrate PAX8 immunoreactivity. Of the gastroenteropancreatic neuroendocrine tumors, tumors of the pancreas and ileocecum most often give rise to metastatic disease at the time of initial presentation. ${ }^{2,29}$ Pape et $a l^{29}$ found that $53 \%$ of pancreatic and $70 \%$ of ileal neuroendocrine tumors demonstrated metastatic disease at initial presentation compared with a $<10 \%$ rate of metastatic disease at presentation for rectal, duodenal, appendiceal, and gastric neuroendocrine tumors. Similarly, Modlin et $a l^{2}$ found that $58 \%$ of small intestinal and $72 \%$ of pancreatic neuroendocrine tumors had non-localized disease at presentation, compared with much lower rate of non-localized disease for other gastrointestinal sites. Thus, a positive PAX8 result would suggest the pancreas as a potential primary site of origin. Although PAX8 immunoreactivity suggests a pancreatic neuroendocrine tumor, it is not entirely specific and other gastrointestinal neuroendocrine tumors, particularly those of the duodenum and rectum and less commonly the stomach, may label with PAX8. Given that neuroendocrine tumors from the rectum, duodenum, and stomach are less likely to present with distant metastatic disease at initial presentation, immunoreactivity for PAX8 will more likely indicate metastatic neuroendocrine tumor from a pancreatic primary.

In the final preparation stages of this current manuscript, a study was published that had very similar methodology, results, and conclusions. ${ }^{30}$ In their study, Long et al found a slightly lower percentage of PAX8-positive primary pancreatic neuroendocrine tumors $(42 / 63,67 \%)$ and metastatic pancreatic neuroendocrine tumors (9/18, 50\%) compared with our study. In addition, Long et al also identified a higher proportion of PAX8-positive rectal neuroendocrine tumors (11/13, 85\%) compared with our analysis (5/17, 29\%). Overall, our study confirms the utility of PAX8 in this setting and expands their data by investigating a broader range neuroendocrine tumors and including metastatic neuroendocrine tumors from primary sites other than the pancreas and terminal ileum. Importantly, our results indicate that PAX8 expression in pancreatic neuroendocrine tumors is retained in metastatic sites of disease.

In current practice, a panel of immunohistochemical markers including CDX-2 and TTF-1 is often used in evaluating for the primary site of origin in well-differentiated neuroendocrine tumors, and PAX8 may be a useful addition to this immunohistochemical panel. Several studies have evaluated the expression of CDX-2 and TTF-1 in pulmonary and gastrointestinal well-differentiated neuroendocrine tumors, although these markers suffer from relatively low sensitivity (TTF-1) and specificity (CDX-2), and thus were not included for evaluation in the current study. Most studies have shown that CDX-2 is strongly and diffusely expressed in most ileal and appendiceal well-differentiated neuroendocrine tumors, with most studies demonstrating little to no staining in gastric, rectal, and pulmonary well-differentiated neuroendocrine tumors. ${ }^{9-11,31-33}$ Relative few studies have included pancreatic tumors in their analysis of CDX-2 in well-differentiated neuroendocrine tumors. Barbareschi et al ${ }^{9}$ demonstrated variable CDX-2 reactivity in 14/48 (29\%) pancreatic well-differentiated neuroendocrine tumors, with staining limited to non-functioning tumors. Schmitt et $a l^{12}$ found 18/87 (20\%) of primary pancreatic neuroendocrine tumors stained with CDX-2, whereas only 1/18 metastatic pancreatic neuroendocrine tumors displayed CDX-2 reactivity. Similarly, Srivastava et al found CDX-2 labeling in only $3 / 31(10 \%)$ primary pancreatic neuroendocrine tumors, although staining was observed in $<50 \%$ of tumor cells. Interestingly, 4/8 (50\%) metastatic pancreatic neuroendocrine tumors in their study demonstrated CDX-2 staining in $>50 \%$ of tumor cells. ${ }^{13}$ Given the overlap of CDX-2 reactivity between midgut well-differentiated neuroendocrine tumors and pancreatic neuroendocrine tumors, particularly in metastatic tumor deposits, CDX-2 immunohistochemistry suffers from relatively low specificity in localizing the primary site of tumor origin. In contrast to CDX-2, TTF-1 immunohistochemistry is relatively specific for pulmonary well-differentiated neuroendocrine tumors, ${ }^{8,10,34-36}$ although rare examples of TTF-1 immunoreactivity in neuroendocrine tumors in the gastrointestinal tract, gynecologic tract, and pancreas $^{12,37}$ have been reported. Oliveira et $a l^{36}$ identified TTF-1 positivity in 27/30 (90\%) primary and metastatic pulmonary well-differentiated neuroendocrine tumors, with no reactivity in pancreatic and intestinal well-differentiated neuroendocrine tumors. Other investigators have shown a lower sensitivity of TTF-1 for pulmonary well-differentiated neuroendocrine tumors. ${ }^{13}$

Recent literature has attempted to expand the panel of immunohistochemical studies that may be used to determine primary site of origin in metastatic well-differentiated neuroendocrine tumors. Schmitt et al ${ }^{12}$ described Islet 1 (Isl1), which labeled $58 / 84(69 \%)$ primary pancreatic neuroendocrine 
tumors and 12/18 (67\%) metastatic pancreatic neuroendocrine tumors. However, Isl1 expression was also seen in well-differentiated neuroendocrine tumors of the lung $(5 / 31,16 \%)$ and ileum $(1 / 30$, $3 \%$ ), indicating that its inclusion in an immunohistochemical panel to determine the primary site of origin of a metastatic well-differentiated neuroendocrine tumors may be of limited utility compared with PAX8, which is uniformly negative in welldifferentiated neuroendocrine tumors of the lung and ileum. More recently, NESP-55 expression was reported in $16 / 39(41 \%)$ primary pancreatic neuroendocrine tumors and not observed in neuroendocrine tumors of other gastrointestinal sites and only rarely in pulmonary well-differentiated neuroendocrine tumors. ${ }^{13}$ Although relatively specific for pancreatic neuroendocrine tumors, NESP suffers from relatively low sensitivity compared with PAX8 immunohistochemistry. Pancreatic neuroendocrine tumors have also been shown to exhibit immunoreactivity with PAX4 (70/96, 73\%) and PAX6 (41/97, 42\%); however, the specificity of these PAX markers for pancreatic neuroendocrine tumors compared with well-differentiated neuroendocrine tumors from other anatomic sites has not been investigated. ${ }^{38}$

Pancreatic neuroendocrine tumors exhibit a broad range of biologic aggressiveness, and the prognosis of these tumors is difficult to predict. In their study, Long et $a l^{30}$ found that PAX8 expression was significantly associated with WHO category 1a compared with $1 \mathrm{~b}$ and 2 and that PAX8-negative tumors were significantly larger and more often metastatic to the liver than PAX8-positive tumors. Our results differ from Long et al and suggest that PAX8 expression is not associated with many clinicopathologic factors associated with biologic aggressiveness, such as WHO categorization, functional status, and the presence of metastatic disease to the liver or lymph nodes. We did identify decreased PAX8 expression in intermediate grade tumors and found that PAX8-negative tumors were more likely to be larger than PAX8-positive tumors; however, these comparisons did not reach statistical significance. Additional studies with a larger number of cases and long-term clinical follow-up would be required to determine the utility of PAX8 as a biomarker of clinical aggressiveness in pancreatic neuroendocrine tumors.

Our study is unique in also evaluating PAX8 expression in solid-pseudopapillary neoplasm and acinar cell carcinoma of the pancreas both of which can be difficult to distinguish from pancreatic neuroendocrine tumors on routine histology. The addition of $\beta$-catenin immunohistochemistry has made the distinction between pancreatic neuroendocrine tumor and solid-pseudopapillary neoplasm easier, as over $90 \%$ of solid-pseudopapillary neoplasms demonstrate abnormal expression of $\beta$-catenin in the nucleus and cytoplasm. ${ }^{39-41}$ In our study, a significant number of solid-pseudopapillary neoplasms demonstrated reactivity with PAX8, which was often weak and focal. Given the overlap of PAX8 expression between pancreatic neuroendocrine tumors and solid-pseudopapillary neoplasm, the addition of PAX8 to an immunohistochemical panel including $\beta$-catenin would not be beneficial. The distinction between acinar cell carcinoma and pancreatic neuroendocrine tumors is important clinically as acinar cell carcinomas typically have a poor prognosis. ${ }^{42}$ Histologically, acinar cell carcinomas and well-differentiated neuroendocrine tumors can be virtually indistinguishable, and the differentiation between these two entities often requires immunohistochemical analysis. Chymotrypsin and trypsin are relatively sensitive immunohistochemical markers of acinar differentiation, ${ }^{43}$ but a significant subset of pancreatic neuroendocrine tumors can focally express these markers. ${ }^{44}$ In our study, none of the acinar cell carcinomas were positive for PAX8. Our results suggest that the addition of PAX8 to a panel of immunohistochemical stains including conventional neuroendocrine markers, synaptophysin and chromogranin, and markers of acinar differentiation, chymotrypsin, and/or trypsin, can help to differentiate between neuroendocrine tumors and acinar cell carcinoma in cases with equivocal histologic features. However, a larger number of cases would need to be studied given the limited number of acinar cell carcinomas included in this study.

Although not typically in the morphologic differential diagnosis of neuroendocrine tumors, immunoreactivity for PAX8 in hematopoietic neoplasms has been described. ${ }^{20}$ PAX8 immunoreactivity may be attributed to cross-reactivity with other members of the PAX gene family, notably PAX5, which is well-characterized marker of B-cells and their associated hematologic malignancies. ${ }^{24}$ Importantly, the recognition of scattered lymphocyte PAX8 reactivity within a neuroendocrine tumor should be emphasized in assessing isolated tumor cell reactivity, particularly in a small biopsy sample.

In summary, PAX8 is expressed in normal pancreatic islet cells and in primary and metastatic pancreatic well-differentiated neuroendocrine tumors. Similar to recently published data, ${ }^{30}$ our results show that PAX8 expression can reliably distinguish pancreatic from ileal and pulmonary well-differentiated neuroendocrine tumors. In addition to pancreatic neuroendocrine tumors, the majority of duodenal neuroendocrine tumors and a subset of rectal, gastric, and appendiceal neuroendocrine tumors may also express PAX8. An immunohistochemical staining panel including PAX8, TTF-1, and CDX-2 would be useful to localize the primary site of tumor involvement by neuroendocrine tumors (Figure 5). Our results indicate that PAX8 immunoreactivity does not have prognostic significance, as no significant difference in PAX8 immunoreactivity was identified between WHO categorization and the presence of metastases, although PAX8-negative 


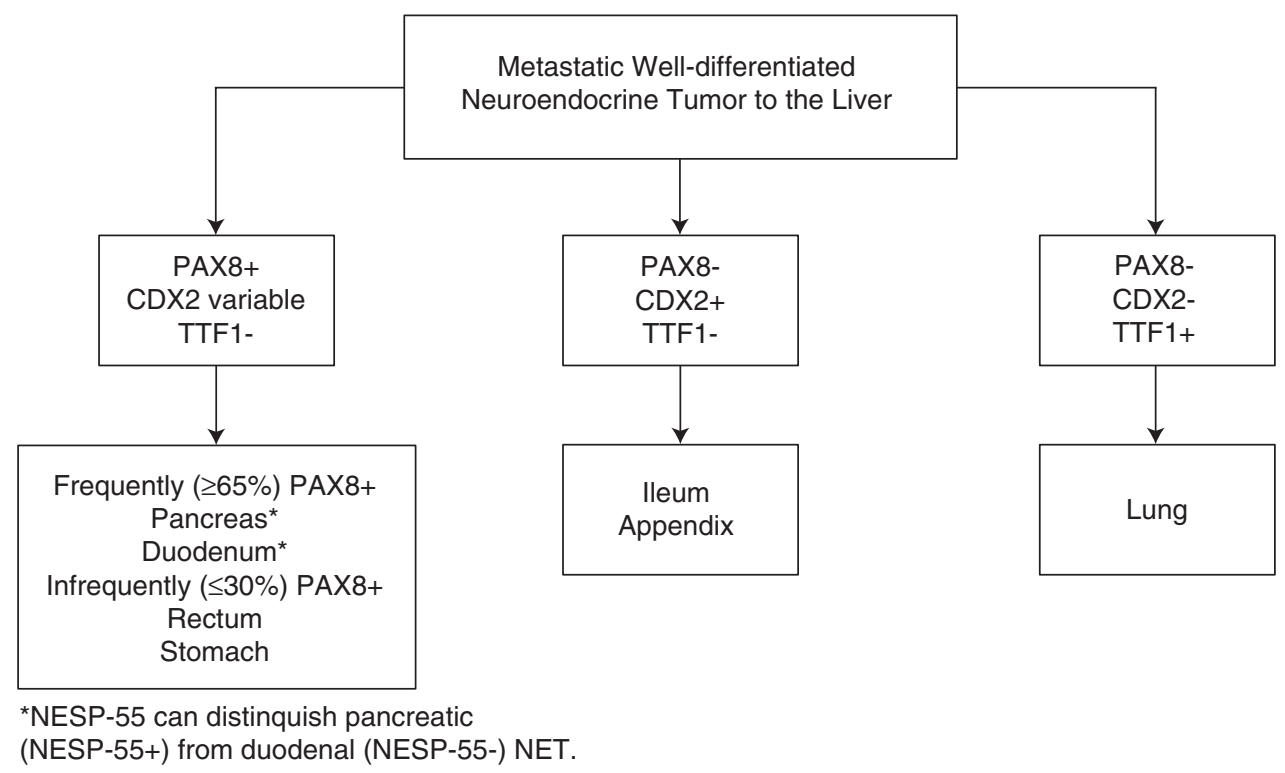

Figure 5 Proposed flowchart to determine the primary site of origin of a metastatic well-differentiated neuroendocrine tumor based on immunohistochemical staining profiles.

pancreatic neuroendocrine tumors tended to be larger in size and were more often intermediate grade. Finally, PAX8 immunohistochemistry may be useful in distinguishing pancreatic neuroendocrine tumors from acinar cell carcinomas, but its utility in distinguishing neuroendocrine tumors from solidpseudopapillary neoplasms is limited, as a significant subset of solid-pseudopapillary neoplasms stain with PAX8.

\section{Disclosure/conflict of interest}

The authors declare no conflict of interest.

\section{References}

1 Gustafsson BI, Kidd M, Modlin IM. Neuroendocrine tumors of the diffuse neuroendocrine system. Curr Opin Oncol 2008;20:1-12.

2 Modlin IM, Lye KD, Kidd M. A 5-decade analysis of 13715 carcinoid tumors. Cancer 2003;97:934-959.

3 Modlin IM, Sandor A. An analysis of 8305 cases of carcinoid tumors. Cancer 1997;79:813-829.

4 Hemminki K, Li X. Incidence trends and risk factors of carcinoid tumors: a nationwide epidemiologic study from Sweden. Cancer 2001;92:2204-2210.

5 Lepage C, Rachet B, Coleman MP. Survival from malignant digestive endocrine tumors in England and Wales: a population-based study. Gastroenterology 2007;132:899-904.

6 Modlin IM, Oberg K, Chung DC, et al. Gastroenteropancreatic neuroendocrine tumours. Lancet Oncol 2008;9:61-72.

7 Yao JC, Hassan M, Phan A, et al. One hundred years after 'carcinoid': epidemiology of and prognostic factors for neuroendocrine tumors in 35825 cases in the United States. J Clin Oncol 2008;26:3063-3072.
8 Cai YC, Banner B, Glickman J, et al. Cytokeratin 7 and 20 and thyroid transcription factor 1 can help distinguish pulmonary from gastrointestinal carcinoid and pancreatic endocrine tumors. Hum Pathol 2001; 32:1087-1093.

9 Barbareschi M, Roldo C, Zamboni G, et al. CDX-2 homeobox gene product expression in neuroendocrine tumors: its role as a marker of intestinal neuroendocrine tumors. Am J Surg Pathol 2004;28:1169-1176.

10 Saqi A, Alexis D, Remotti F, et al. Usefulness of CDX2 and TTF-1 in differentiating gastrointestinal from pulmonary carcinoids. Am J Clin Pathol 2005;123:394-404.

11 Jaffee IM, Rahmani M, Singhal MG, et al. Expression of the intestinal transcription factor CDX2 in carcinoid tumors is a marker of midgut origin. Arch Pathol Lab Med 2006;130:1522-1526.

12 Schmitt AM, Riniker F, Anlauf M, et al. Islet 1 (Isl1) expression is a reliable marker for pancreatic endocrine tumors and their metastases. Am J Surg Pathol 2008;32:420-425.

13 Srivastava A, Hornick JL. Immunohistochemical staining for CDX-2, PDX-1, NESP-55, and TTF-1 can help distinguish gastrointestinal carcinoid tumors from pancreatic endocrine and pulmonary carcinoid tumors. Am J Surg Pathol 2009;33:626-632.

14 Turque N, Plaza S, Radvanyi F, et al. Pax-QNR/Pax-6, a paired box- and homeobox-containing gene expressed in neurons, is also expressed in pancreatic endocrine cells. Mol Endocrinol 1994;8:929-938.

15 Ritz-Laser B, Estreicher A, Gauthier B, et al. The paired homeodomain transcription factor Pax-2 is expressed in the endocrine pancreas and transactivates the glucagon gene promoter. J Biol Chem 2000;275: 32708-32715.

16 Stuart ET, Kioussi C, Gruss P. Mammalian Pax genes. Annu Rev Genet 1994;28:219-236.

17 Tong GX, Yu WM, Beaubier NT, et al. Expression of PAX8 in normal and neoplastic renal tissues: an immunohistochemical study. Mod Pathol 2009;22: 1218-1227. 
18 Nonaka D, Chiriboga L, Soslow RA. Expression of pax8 as a useful marker in distinguishing ovarian carcinomas from mammary carcinomas. Am J Surg Pathol 2008;32:1566-1571.

19 Nonaka D, Tang Y, Chiriboga L, et al. Diagnostic utility of thyroid transcription factors Pax8 and TTF-2 (FoxE1) in thyroid epithelial neoplasms. Mod Pathol 2008;21:192-200.

20 Sangoi AR, West RB, Bonventre JV, et al. Exploring the specificity of putative renal cell carcinoma (RCC) markers in non-renal tissues and neoplasms from various organ systems: a tissue microarray (TMA) study of 501 cases [abstract]. Mod Pathol 2010; 23:216A.

21 Solcia E, Kloppel G. WHO: Histological Typing of Endocrine Tumors. IARC Press: Lyon, France, 2000.

22 Hochwald SN, Zee S, Conlon KC, et al. Prognostic factors in pancreatic endocrine neoplasms: an analysis of 136 cases with a proposal for low-grade and intermediate-grade groups. J Clin Oncol 2002;20: 2633-2642.

23 Beasley MB, Thunnissen FB, Hasleton PS, et al. Carcinoid Tumor. IARC Press: Lyon, France, 2004.

24 Morgenstern DA, Hasan F, Gibson S, et al. PAX5 expression in nonhematopoietic tissues. Reappraisal of previous studies. Am J Clin Pathol 2010;133: 407-415.

25 Perez EA, Koniaris LG, Snell SE, et al. 7201 carcinoids: increasing incidence overall and disproportionate mortality in the elderly. World J Surg 2007;31: 1022-1030.

26 Kulke MH, Hornick JL, Frauenhoffer C, et al. O6methylguanine DNA methyltransferase deficiency and response to temozolomide-based therapy in patients with neuroendocrine tumors. Clin Cancer Res 2009; 15:338-345.

27 Kulke MH, Stuart K, Enzinger PC, et al. Phase II study of temozolomide and thalidomide in patients with metastatic neuroendocrine tumors. J Clin Oncol 2006; 24:401-406.

28 Mansouri A, St-Onge L, Gruss P. Role of genes in endoderm-derived organs. Trends Endocrinol Metab 1999;10:164-167.

29 Pape UF, Bohmig M, Berndt U, et al. Survival and clinical outcome of patients with neuroendocrine tumors of the gastroenteropancreatic tract in a german referral center. Ann N Y Acad Sci 2004;1014: 222-233.

30 Long KB, Srivastava A, Hirsch MS, et al. PAX8 Expression in well-differentiated pancreatic endocrine tumors: correlation with clinicopathologic features and comparison with gastrointestinal and pulmonary carcinoid tumors. Am J Surg Pathol 2010;34:723-729.

31 Erickson LA, Papouchado B, Dimashkieh H, et al. $\mathrm{Cdx} 2$ as a marker for neuroendocrine tumors of unknown primary sites. Endocr Pathol 2004;15: 247-252.
32 La Rosa S, Rigoli E, Uccella S, et al. CDX2 as a marker of intestinal EC-cells and related well-differentiated endocrine tumors. Virchows Arch 2004;445:248-254.

33 Lin X, Saad RS, Luckasevic TM, et al. Diagnostic value of CDX-2 and TTF-1 expressions in separating metastatic neuroendocrine neoplasms of unknown origin. Appl Immunohistochem Mol Morphol 2007;15: 407-414.

34 Agoff SN, Lamps LW, Philip AT, et al. Thyroid transcription factor-1 is expressed in extrapulmonary small cell carcinomas but not in other extrapulmonary neuroendocrine tumors. Mod Pathol 2000;13:238-242.

35 Folpe AL, Gown AM, Lamps LW, et al. Thyroid transcription factor-1: immunohistochemical evaluation in pulmonary neuroendocrine tumors. Mod Pathol 1999;12:5-8.

36 Oliveira AM, Tazelaar HD, Myers JL, et al. Thyroid transcription factor-1 distinguishes metastatic pulmonary from well-differentiated neuroendocrine tumors of other sites. Am J Surg Pathol 2001;25:815-819.

37 McCluggage WG, Kennedy K, Busam KJ. An immunohistochemical study of cervical neuroendocrine carcinomas: neoplasms that are commonly TTF1 positive and which may express CK20 and P63. Am J Surg Pathol 2010;34:735-741.

38 Zhang L, Smyrk TC, Oliveira AM, et al. KIT is an independent prognostic marker for pancreatic endocrine tumors: a finding derived from analysis of islet cell differentiation markers. Am J Surg Pathol 2009; 33:1562-1569.

39 Abraham SC, Klimstra DS, Wilentz RE, et al. Solidpseudopapillary tumors of the pancreas are genetically distinct from pancreatic ductal adenocarcinomas and almost always harbor beta-catenin mutations. Am J Pathol 2002;160:1361-1369.

$40 \mathrm{Kim}$ MJ, Jang SJ, Yu E. Loss of E-cadherin and cytoplasmic-nuclear expression of beta-catenin are the most useful immunoprofiles in the diagnosis of solid-pseudopapillary neoplasm of the pancreas. Hum Pathol 2008;39:251-258.

41 Tang LH, Aydin H, Brennan MF, et al. Clinically aggressive solid pseudopapillary tumors of the pancreas: a report of two cases with components of undifferentiated carcinoma and a comparative clinicopathologic analysis of 34 conventional cases. Am J Surg Pathol 2005;29:512-519.

42 Holen KD, Klimstra DS, Hummer A, et al. Clinical characteristics and outcomes from an institutional series of acinar cell carcinoma of the pancreas and related tumors. J Clin Oncol 2002;20:4673-4678.

43 Klimstra DS, Heffess CS, Oertel JE, et al. Acinar cell carcinoma of the pancreas. A clinicopathologic study of 28 cases. Am J Surg Pathol 1992;16:815-837.

44 Yantiss RK, Chang HK, Farraye FA, et al. Prevalence and prognostic significance of acinar cell differentiation in pancreatic endocrine tumors. Am J Surg Pathol 2002;26:893-901. 